This item was submitted to Loughborough's Research Repository by the author.

Items in Figshare are protected by copyright, with all rights reserved, unless otherwise indicated.

\title{
Computer-supported collaborative accounts of major depression: digital rhetoric on Quora and Wikipedia
}

PLEASE CITE THE PUBLISHED VERSION

https://doi.org/10.1109/CISTI.2014.6876968

PUBLISHER

(C) IEEE

VERSION

AM (Accepted Manuscript)

LICENCE

CC BY-NC-ND 4.0

\section{REPOSITORY RECORD}

Rughinis, Cosima, Bogdana Huma, S. Matei, and Reiner Rughinis. 2019. "Computer-supported Collaborative Accounts of Major Depression: Digital Rhetoric on Quora and Wikipedia”. figshare.

https://hdl.handle.net/2134/33506. 


\title{
Computer-Supported Collaborative Accounts of Major Depression
}

\author{
Digital Rhetoric on Quora and Wikipedia
}

\author{
Cosima Rughiniş, Bogdana Humă, Ștefania Matei \\ Department of Sociology \\ University of Bucharest \\ Bucharest, Romania \\ \{cosima.rughinis, bogdana.huma, \\ stefania.matei\}@sas.unibuc.ro
}

\author{
Răzvan Rughiniș \\ Department of Computer Science \\ University of Bucharest \\ Bucharest, Romania \\ razvan.rughinis@cs.pub.ro
}

\begin{abstract}
We analyze digital rhetoric in two computersupported collaborative settings of writing and learning, focusing on major depression: Wikipedia and Quora. We examine the procedural rhetoric of access to and interaction with information, and the textual rhetoric of individual and aggregated entries. Through their different organization of authorship, publication and reading, the two settings create divergent accounts of depression. Key points of difference include: focus on symptoms and causes vs. experiences and advice, use of lists vs. metaphors and narratives, a/temporal structure, and personal and relational knowledge.
\end{abstract}

Keywords-digital rhetoric; computer supported collaborative knowledge making; major depression; Quora; Wikipedia

\section{INTRODUCTION}

Readers interested in learning about depression can find on the Internet a lot of forms of knowledge about this condition. There are organizational and personal sites and blogs, online books, as well as various collaborative settings such as forums, wikis, and Q\&A communities.

Previous research has investigated the functioning of collaborative knowledge-making sites, looking into contributors' practices and motivation, on the social side, and specific technologies for relating questions to answerers, identifying quality in contributions and trustworthiness in answerers, among others, on the technical side [1]-[3]. With rare exceptions, analyses of social Q\&A sites, wikis, and other collaborative inquiry platforms is usually topic-indifferent, examining contributions in the aggregate, across multiple subjects (but see articles on history writing in Wikipedia [4] and biographical writing in Wikipedia [5]).

We are interested in understanding the topic-specific influence of social Q\&A sites, as regards mental illness. Specifically, we examine the rhetorical distinctiveness of Quora and Wikipedia, and the consequences of their social organization on the construct of major depression, as it is rendered through their contributors.

Through our approach, we also contribute to the discussion of information quality in collaborative online knowledgemaking. Information quality is often conceptualized in terms of metrics such as: completeness, accuracy, verifiability, timeliness, and reliance on sources [1]. We are particularly interested in how social sites of knowledge-making solve the unavoidable conflicts between various sources, perspectives, voices - thus achieving 'completeness' and 'reliance on sources' in specific ways, with various degrees of standardization, unification or diversity of perspectives, formalization or personalization of voices [6]. Different collaborative authorship settings give rise to distinctive genres (ibid.) - which, in turn, lead to different constructs of mental conditions.

Maybe more than questions concerning the non-human natural world, knowledge about human experiences is genredependent - that is, constructs of 'love', 'sadness', 'revolution' or, for that matter, 'major depression', can be rendered intelligible in very different ways. Such constructs are rhetorically constituted by authors for their audiences in specific settings. We compare Wikipedia and Quora (similarly to [6]) in order to make visible the rhetorical properties of these two communities of authorship, publication and reading, and their consequences on knowledge creation about depression.

In brief, we address the following research questions:

(1) How do contributors assemble renditions of depression through specific forms of organizing authorship, publication and reading, in online collaborative settings and through distinctive rhetorical methods?

(2) How does the construct of depression on Quora differ from the construct of depression on Wikipedia?

The paper is organized as follows: we first discuss the relevance of the organization of (hyper-)textual writing, publishing and reading for the construct of mental illness. We then present the organization of practices in the two online settings. We go on to describe the structures of the depression constructs, as highlighted through content analysis. The final section concludes the paper.

\section{RHETORICAL CONSTRUCTION OF MENTAL ILLNESS}

In daily life, people affected by depression or other psychological conditions often seem, to others, to display a 
kind of disorder. Starting from Smith's discussion of the construct of mental illness rendered in an interview [7], as lay persons we present and observe mental illness in stories and other accounts through a specific organization of the text, which instructs us how to make sense of a collection of instances of atypical behavior. Smith concludes that mental illness is conveyed, rhetorically, as a disordered collection of disorderly actions - a list of instances of misbehavior of which the typical reader cannot make any sense. The disorder of 'mental disorders' is rhetorically communicated through an accumulation of misfitting actions (ibid.).

The diagnosis of a psychological condition brings order to chaos - enabling observers to make sense of the persons' actions, to reinterpret them or at least to explain them through an underlying, unifying cause. The action of learning about a psychological condition means that the learner simultaneously acquires information about (1) the behavioral disorder and (2) the explanatory ordering device. Learning about mental illness is, in a way, paradoxical, as we must at the same time grasp real chaos and real underlying order. We understand mental illnesses by becoming familiarized with an array of atypical behaviors and with accounts through which we reinterpret them as orderly-after-all.

Order and chaos are conveyed not just strictly through explicit descriptions, but also through the organization of information in a certain text (or, in our case, hypertext). We are interested in how different online collaborative settings create experiences of order and chaos that afford a specific understanding of mental illness.

When studying collaboratively authored accounts of depression in online settings, we examine two types of rhetoric. On the one hand, there is the digital rhetoric [8] of online texts, in which we find different uses of Aristotle's three resources logos, pathos, and ethos:

- The rhetoric of posts examined individually or in the aggregate;

- The rhetoric of collections of posts, examined through their diversity and the relationships between posts.

On the other hand, online collaborative platforms engage readers in various interactions with texts and with other users, through procedures of access, contribution, review, voting, commenting etc. Bogost argues that we should also examine procedural rhetoric [9] - the persuasive impact of procedures in games, software or, in our case, knowledge-making platforms. From this perspective, we should inquire into the rhetorical effects of several procedures on the constitution of 'major depression':

- $\quad$ Typical journeys of readers looking to find knowledge on depression: how are pages found?

- Typical interactions of readers with the hyper-text and platform software options;

- Typical interactions of readers with authors and other participants.

In order to understand the distinctive rhetoric of text and procedures in Quora and Wikipedia, we discuss in the next section their organization of authorship, publication, and reading.

\section{ORGANIZATION OF KNOWLEDGE PRODUCTION SETTINGS: WIKIPEDIA AND QUORA}

Both Wikipedia and Quora rely on collaborative authorship, but the two platforms differ systematically in their organization and the resulting genres of textual knowledge.

Wikipedia is an online encyclopedia, edited by a collective of collaborators usually working under pseudonyms, guided by Wikipedia's rules and criteria of good content. Experienced users have an important role in establishing the final versions of an entry, as they master better than newcomers the procedures for writing encyclopedic content and the art of argumentation based on these procedures [5], [10], [11]. Wikipedia has been criticized for its anti-elitism [12], and it stands at the forefront of discussions concerning the emergence of new types of expertise, based on dialogue among large numbers of interested contributors [13], [14]. Wikipedia editorial policies put a large weight on properly using and referencing sources, favoring scientific sources and other reputable materials. The encyclopaedic voice is expected to represent controversies according to their public visibility and significance, while keeping an unified style and a balanced perspective - the so-called Neutral Point of View [15], [16]

Wikipedia offers readers several types of information, clearly separated on different pages:

- Article pages, for example the entry on Major depressive disorder [17];

- Talk pages, on which readers can follow complete discussions among editors and their decisions; talk page archives are often long, and readers are unlikely to read them unless they have a very specific interest in a modification. Talk pages represent the backstage of Wikipedia's entries;

- $\quad$ The history of the page, listing all previous versions.

The typical trajectory of a Wikipedia reader involves a Google search on the required key words, which often leads to Wikipedia as a top link (as it is the case for googling 'depression', which, at least in our searches, points to Wikipedia entries on 'Depression (mood)' and 'Major depressive disorder'.) Readers can also search through Wikipedia's engine, which points to a disambiguation page differentiating depression in a psychological sense (actually listed under Medicine) from those belonging to Earth science and Economics (Figure 1. )

Quora, unlike Wikipedia, is a Q\&A site, conducive to a different organization of work. Quora requires members to register with their real names, although anonymous posting is allowed and is quite frequent on sensitive topics such as mental illness. Quora has attracted attention and popularity through its claims to offer experiential expertise [3] - answers based in personal, direct engagement of answerers' with the topic, certifiable through their CV's, since they are not anonymous. Quora combines the Q\&A infrastructure with a social network 
similar to Twitter, based on asymmetric following, which plays an important role in matching questions to answerers (ibid.).

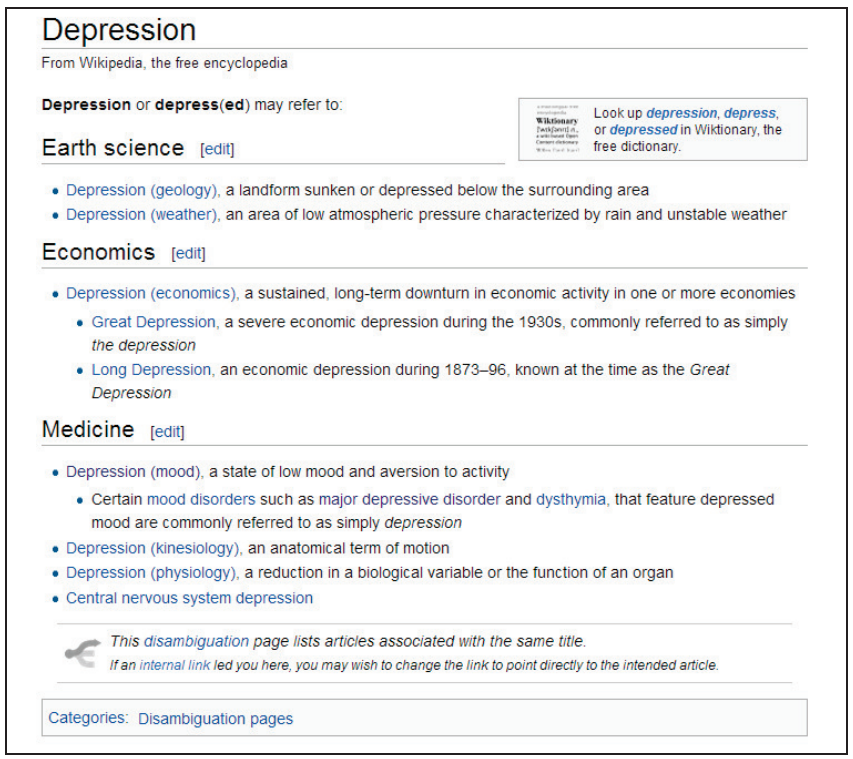

Figure 1. The search / disambiguation page of Depression in Wikipedia

Quora members have two ways of accessing content: (1) through direct search for a key word, or (2) through their personalized feed, based on the topics, questions, and authors that they follow. When searching for 'depression', readers are presented with search results such as in Figure 2., pointing to the 'Depression' topic, as well as to numerous questions already asked about depression. Each question is illustrated with a few lines from the top ranked answer and with the count of its upvotes.

The reader can click on any question, gaining access to the question page. Here, all answers are presented sequentially. The default view is the so-called 'Magic': answers are ranked by Quora's algorithm, which relies on upvotes related to the number of views, among others (the algorithm is not public) (see Figure 3. ). Readers can see, for each question page:

- The full question text, and related questions;

- Author's name and a self-styled description establishing specific expertise or, more often, offering a personal motto;

- Total number of answers;

- The number of upvotes for each answer, and, below the answer text, the number of comments and sharing operations; readers should click on comments to read them or post any. Comments are not part of the main question page, and are considerably less frequent than upvotes.

Readers can also opt for ranking answers in terms of votes or date of posting. From each answer, the reader can access its readers' comments, if any, and can click on its author's profile (if it was not posted anonymously). The reader may also go to the "Depression" topic ${ }^{1}$, thus accessing all questions labeled with this concept, and related topics.

Quora depression
Results for "depression"
Depression
Depression may be described as feeling sad, blue, unhappy, miserable, or down
in the dumps. Most of us feel this way at one time or another for short periods. True
clinical... (more)
Topic: Top Stories . Questions
* What does it feel like to have depression?
Anonymous - In the middle of Los Angeles, California there is an area called the
La Brea Tar Pits, where asphalt and crude oil seep up to form pools on the surface
of the ground. There... (more)
What are the biggest misconceptions about depression?
Jane Chin, Lived to Tell. - Phase I.- Before Diagnosis ... That I didn't apply
myself enough to snap out of it. * That I had too many things going for me to be
depressed. *That I was simply too sensitive for... (more)
What positives has depression added to your life?
Jane Chin, Lived to Tell. - LABELING what I experienced all my life as "major
depressive disorder" ("depression") has been the single-most "major positive
development" in my life.... Hey! So, I'm not a ... (more)
* What is the evolutionary advantage of depression?
Vilan Natanzon, Watched hundreds of ho... (more) — http.//www.theatlantic.co
m/magaz.... An interesting article about genes that cause depression. Rhesus
monkeys are the only ones apart from us with these genes and they're s... (more)
What is it like to be in a relationship with someone with
depression?
Anonymous — Last week I ended a 2-year relationship with the girl I loved, partly
as a result of the depression she's been battling most of her life and through all of
our relationship.... (more)

Figure 2. The search page of Depression in Quora

Depression: Mental Health Psychology What Does It Feel Like to X? Edit
* What does it feel like to have depression? Edit
Sub question: is depression the same as bipolar disorder?
Also see http://www.quora.com/What-do-cli...
Edit
3 Comments - Share (5) - Report - Options
89 Answers
Anonymous
Votes by Cosima Rughinis, Mani Cavalieri, David Dreshfield, Bevan Audstone, and 406
more.
In the middle of Los Angeles, California there is an area called the La Brea Tar Pits,
where asphalt and crude oil seep up to form pools on the surface of the ground. There is
a museum nearby where one can view the fossilized remains of animals, from beetles to
mastodons, that became stuck in the tar forever over thousands of years, with an exhibit
where one can experience just how much strength it would take to escape the pit. The
pools have an unpleasant smell and appear faintly menacing, a murky brown-black with
an insectoid petroleum sheen.
Suffering from major depression feels like sinking into a tar pit.

Figure 3. Experiential knowledge: 'What does it feel to have depression?' in Quora

\section{RHETORICAL CONSTRUCTION OF MAJOR DEPRESSION ON QUORA AND WIKIPEDIA}

Starting from a background understanding of how these two platforms function, we attempt to identify the main

\footnotetext{
${ }^{1} \mathrm{http}: / /$ www.quora.com/Depression
} 
differences in the resulting construct of major depression, and to trace their origins.

Several points of divergence are clearly visible in the above presentation, especially concerning formality and standardization [6]. Wikipedia promotes formal, standardized formulations, making use of logos rather than pathos, and establishing credibility (ethos) through references to authoritative, preferably scientific materials. Wikipedia pages are written in a single, quite homogenous and impersonal voice, in charge of balancing conflicting viewpoints and managing uncertainties. On the contrary, Quora search pages and question pages are multivocal and informal, combining accounts from various authors, often written in different styles, and with various personal profiles. While Wikipedia editors' expertise refers especially to understanding scientific accounts of depression and mastering editing procedures and argumentation skills on the Talk page, Quora's contributors, more often than not, assert their own experiential knowledge of depression: they have been depressed or have lived in a close relationship with a depressed person.

TABLE I. DIVERGENT RHETORICAL CONSTRUCTIONS

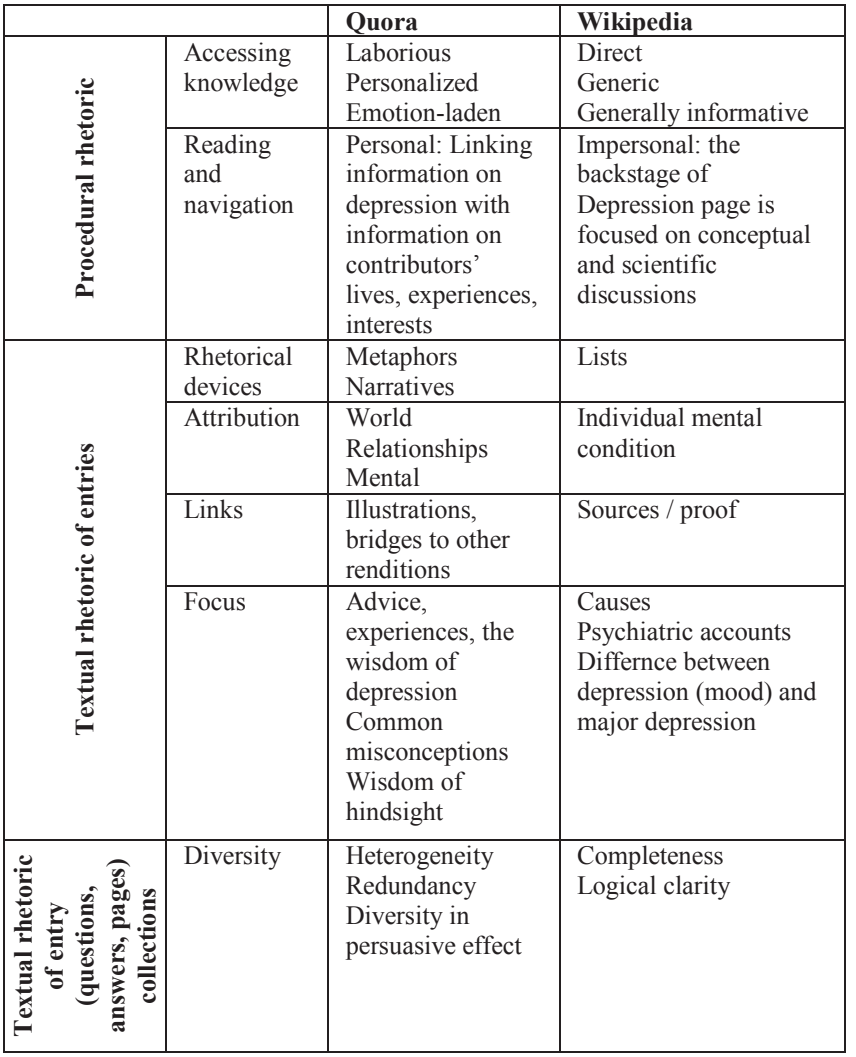

In order to identify specific rhetorical operations on the two platforms, we examine separately the procedural and the textual devices at work (see TABLE I. )

\section{A. Procedural rhetoric of Quora and Wikipedia}

The diverging journeys of the reader in search for information on the two platforms is consequential for the constitution of the depression construct.
Wikipedia's pages are easily and directly accessible. The only challenge is to choose between two different pages that refer to depression - namely the mood and the mood disorder. The distinction between depression as a frequent but reversible mood and major depression as a condition of clinical significance is very important for Wikipedia contributors, who discuss it at length. Interestingly, it plays virtually no role on Quora, where participants ask and answer about depression, occasionally distinguishing minor from major versions, but mostly relying on an implicit consensus that a grave condition is at stake. Terminological accuracy is often a concern on Wikipedia, but less so on Quora - at least as regards depression.

On the contrary, the reader seeking information on depression on Quora has to work to choose questions, select answers, evaluate whether the answer is satisfactory and deciding to read more or move to something else. Information on Quora must be actively pursued, and this work generates emotions: readers may find some answers interesting, moving, well argued or somehow else deeply satisfying - while others are experienced as redundant, inadequate, trolling, simplistic etc. In the end, the reader will probably find several answers which constitute her / his preferred accounts of depression. Quora stimulates the assembly of a personal body of knowledge, adapted to one's interests and rhetorical preferences. (Interestingly, there is no possibility of actually saving preferred answers in a personal folder or a similar technical way of personalization). Users are also invited to vote and comment, thus engaging them more directly with what they read. Understanding depression becomes, thus, a cognitive adventure.

Wikipedia also invites readers to edit entries; still, only a minority of users takes on this challenge. By design, editing is less about one's own experiences, but about one's capacity to assemble an objectively-phrased, common knowledge on the topic.

The depression construct on Wikipedia is impersonal, presented by the encyclopaedic voice from a certain distance that allows broad coverage of scientific topics, systematization, and balance. On the contrary, the depression construct on Quora is personal: the reader navigates from answers about depression to personal profiles of authors and their other activity on site, including other answer posted - which, more often than not, refer to completely different topics. Depression is directly experienced as a part of people's lives, as a critical condition while at the same time not exhausting their activity at least, not at present time.

Due to the laborious and whimsical trajectory of seeking and reading Quora information about depression, there is no obvious structure of this knowledge available for any reader. Each person will be exposed to a certain corpus of questions and answers, depending on her / his choices within available options. On the contrary, the Wikipedia page provides a clear overview of the topic through its table of contents (see Figure 4. ) - thus offering the reader an easy option of looking for an additional topic.

Still, it is likely that the experience with the structure of the construct will also differ from Quora users to Wikipedia 
readers. We have assembled a list with the top 60 questions presented on the search page for 'Depression' key word (on 21.02.2014) and we have classified them into 10 types: solutions (asking for advice, asking about others' solutions); the experience of depression (how does it feel like); positives (the wisdom of depression); relations (how to relate with depressed others, or how people relate with others when depressed); misconceptions about depressions; use of medication; various curiosity questions (is depression a modern disease? does humor help?); causes of depression; signs and symptoms of depression, and, finally, good readings about depression. Figure 5. presents the distribution of the top 60 questions on these types, according to the number of questions and also according to the number of answers received by each question. While the number of questions per type varies moderately, the distribution of answers is strongly differentiated.

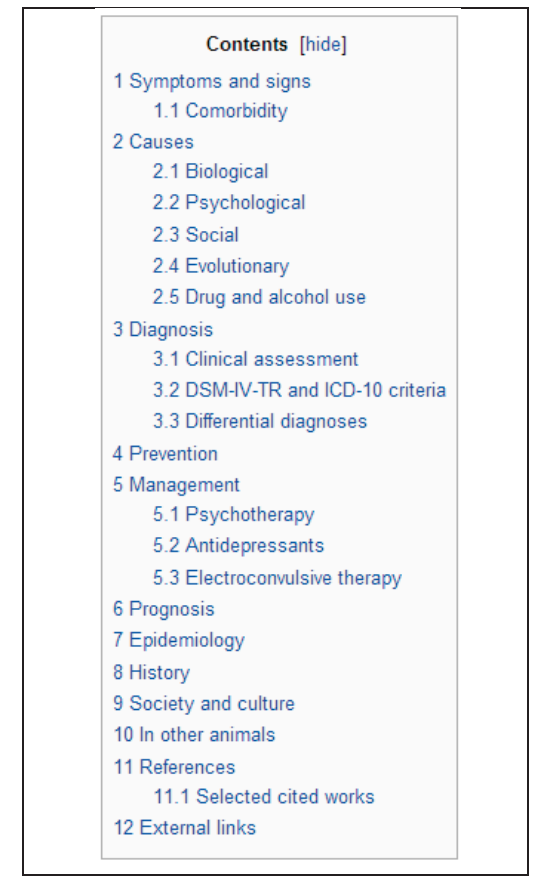

Figure 4. Thematic structure: The table of contents for the Wikipedia entry on Major Depressive Disorder

The most important concerns of Quora members are: advice and solutions regarding depression, the experience of depression, and the positive side of it. Depression causes and lists of signs and symptoms are much less frequent in questions and answers as well. On the contrary, the first two sub-sections of the Wikipedia entry on major depression are Symptoms and signs, respectively Causes.

\section{B. Digital rhetoric of hyper-texts in Quora and Wikipedia}

There are significant differences between Quora and Wikipedia not only as regards users' activities when seeking for information, but also between writing styles in individual entries, and properties of entry collections (TABLE I. ).

A large part of Quora answers about depression consist in retrospective accounts (thus differentiating it from forums on which currently depressed persons present their thoughts and problems). Quora constructs depression with the wisdom of hindsight - while Wikipedia makes use of psychiatric concepts and classifications as its main knowledge resource.

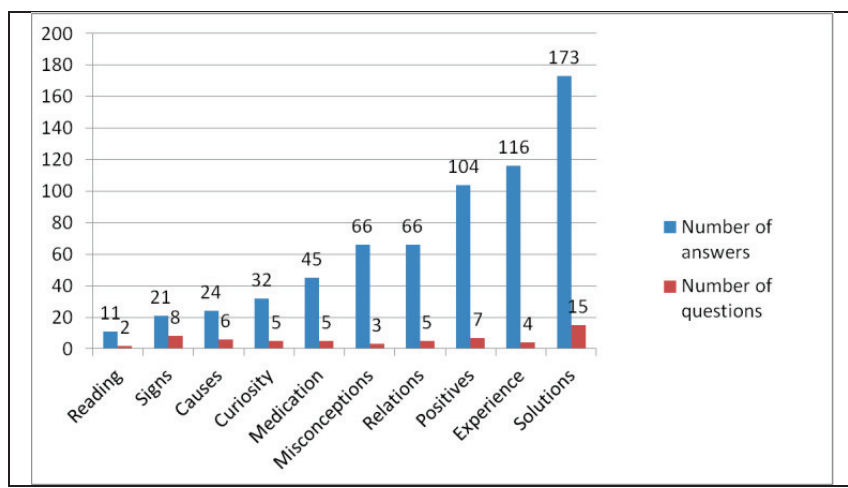

Figure 5. Thematic structure: Classification of questions in the 'Depression' search page (authors' analysis of first 60 questions), Quora, 21.02.2014

While links in the Wikipedia article consist in sources that act as proof for entry statements, links in Quora posts often serve as 'bridges' to experiences in different genres, including cartoons [18], [19], visual arts [20] and computer games [21].

One of the most important rhetorical devices on Wikipedia's rendition of major depression is the list. For example, depression symptoms are presented in a list:

"A person having a major depressive episode usually exhibits a very low mood, which pervades all aspects of life, and an inability to experience pleasure in activities that were formerly enjoyed. Depressed people may be preoccupied with, or ruminate over, thoughts and feelings of worthlessness, inappropriate guilt or regret, helplessness, hopelessness, and self-hatred" [17].

Lists are also used for discussing the affected aspects of life, the possible causes, neurological risk conditions, or diagnosis information. Lists and logical classifications are part of the rhetorical reliance on logos, to achieve rationality and completeness as visible and defining features of the encyclopaedic article.

Quora entries make often use of metaphors and narratives. The top answer to "How does it feel like to have depression", argues that "Suffering from major depression feels like sinking into a tar pit" (Anonymous). The second answer asks: "Ever tug at a loose yarn and watch the whole piece unravel?" (Mike Xie); the sixth answer, as of 21.02.2014, likens depression to a tapeworm (Mani Cavalieri). Still, the list is also an important device on Quora: the third and seventh answers (ranked on 21.02.2014) have the form of bulleted lists, among others. While this is one of the rhetorical options on this platform, it is the dominant structure on Wikipedia.

Metaphors afford novel understandings of depression. By analogy with a "tar pit", or with the "undertow of the ocean" (Anonymous), one can represent depression as an environment,

${ }^{2}$ http://www.quora.com/Depression/What-does-it-feel-like-tohave-depression 
a world that is external to the person - rather than an individual, internal, mental condition:

"Metaphorically speaking... It's like the undertow of the ocean. It seems innocuous at first: I can handle the waves; it's not that deep; I can get myself back to shore; I am strong enough. You continue living your life, playing your part, going through the motions of living freely, ignoring the dynamics of the pull, and deluding yourself into believing your status is unchanged. (...) It is invisible. It is silent. It is massive. It wants you to stay with It out in the cold depths where you can't touch and your strokes toward the shore yield so little progress relative to the herculean effort you exert trying to get back to your former status quo. You struggle to overcome Its force, knowing that there are people and things on the shore needing your attention. You buckle down, throwing everything you have into the effort to return. When you finally look up ahead to chart your progress, you are dismayed to see how little distance you have seemingly covered, but something else jars your mind: the shore. It's changed. Almost imperceptibly. The people are still there, laughing, playing, picnicking, coming, and going. But no one is looking for you! They've continued their lives without you. No search party. No emergency. Nothing." (Anonymous)

A frequent topic on Quora answers also refers to relationships - the need for support, sometimes unmet, and the burdens on family and close ones; the impossibility of understanding depression from a non-depressed state; communication with a depressed person. This relational focus is virtually absent from the Wikipedia construct.

The narrative structure of many Quora answers points to trajectories of "sinking" and redemption, as well as recurrent episodes. The very existence of hindsight answers about depression is a witness to the possibility of healing - a living proof to the temporal structure of depression as something that ebbs and flows across people and periods. In contrast, the Wikipedia presentation is a-temporal - situated in the abstract present tense of scientific accounts. Its only past tense is historical (in the 'History' subsection).

\section{CONCLUSIONS: DIVERGING CONSTRUCTS OF MAJOR DEPRESSION ON QUORA AND WIKIPEDIA}

Our analysis highlights significant differences between the constructs of depression assembled through questions and answers on Quora, respectively through collaborative editing on Wikipedia. The two platforms serve different purposes, reflected in distinctive writing genres. The procedural and textual rhetoric of these genres lead to divergent renditions of major depression in the two online settings.

Our analysis proposes a schema for analyzing constructs of mental illness in collaborative knowledge-making sites (see TABLE I. ). Such a tool could be useful both for students in psychology and psychiatry and for students in information science, pointing to the close relationships between technologies, social organization of authorship, and representations of mental illness.

\section{ACKNOWLEDGMENT}

This article has been supported by the research project "Sociological imagination and disciplinary orientation in applied social research", with financial support of ANCS / UEFISCDI with grant no. PN-II-RU-TE-2011-3-0143, contract no. $14 / 28.10 .2011$.

\section{REFERENCES}

[1] R. Gazan, "Social Q\&A," J. Am. Soc. Inf. Sci. Technol., vol. 62, no. 12, pp. 2301-2312, 2011.

[2] S. A. Paul, L. Hong, and E. H. Chi, "Who is Authoritative? Understanding Reputation Mechanisms in Quora," in Collective Intelligence, 2012, pp. 1-8.

[3] G. Wang, K. Gill, M. Mohanlal, H. Zheng, and B. Y. Zhao, "Wisdom in the Social Crowd: An Analysis of Quora," in International World Wide Web Conference, 2013, pp. 1341-1352.

[4] R. Rosenzweig, "Can History Be Open Source? Wikipedia and the Future of the Past," J. Am. Hist., vol. 93, no. 1, pp. 117-146, 2006.

[5] C. Rughiniș and Ștefania Matei, "Learning Through Massively CoAuthored Biographies. Making Sense of Steve Jobs on Wikipedia through Delegated Voice," in The 19th International Conference on Control Systems and Computer Science CSCS19, 2013, pp. 1-7.

[6] W. Emigh and S. C. Herring, "Collaborative Authoring on the Web: A Genre Analysis of Online Encyclopedias," in Proceedings of the ThirtyEighth Hawai'i International Conference on System Sciences (HICSS38), 2005, pp. 1-10.

[7] D. E. Smith, "K. is Mentally Ill: The Anatomy of a Factual Account," in in Texts, Facts, and Femininity. Exploring the Relations of Ruling, New York, NY: Routledge, 1990, pp. 9-40.

[8] J. P. Zappen, "Digital Rhetoric: Toward an Integrated Theory," Tech. Commun. Q., vol. 14, no. 3, pp. 319-325, 2005.

[9] I. Bogost, "The Rhetoric of Video Games," in in The Ecology of Games: Connecting Youth, Games, and Learning, K. Salen, Ed. Cambridge: MIT Press, 2008, pp. 117-140.

[10] L. Xiao and N. Askin, "Deliberation in Wikipedia: Rationales in article deletion discussions," Proc. Am. Soc. Inf. Sci. Technol., vol. 49, no. 1, pp. 1-4, Jan. 2012.

[11] B. Luyt, "The inclusivity of Wikipedia and the drawing of expert boundaries: An examination of talk pages and reference lists," $\mathrm{J}$. Am. Soc. Inf. Sci. Technol., vol. 63, no. 9, pp. 1868-1878, Sep. 2012.

[12] L. Sanger, "Why Wikipedia Must Jettison Its Anti-Elitism," Kuro5hin, no. 30.12.2004, 2004.

[13] E. J. Hartelius, "Wikipedia and the Emergence of Dialogic Expertise," South. Commun. J., vol. 75, no. 5, pp. 505-526, Nov. 2010.

[14] D. Fallis, "Toward an epistemology of Wikipedia," J. Am. Soc. Inf. Sci. Technol., vol. 59, no. 10, pp. 1662-1674, Aug. 2008.

[15] Wikipedia Contributors, "Wikipedia: Neutral Point of View," Wikipedia, The Free Encyclopedia, 2013. [Online]. Available: http://en.wikipedia.org/wiki/Wikipedia:Neutral_point_of_view. [Accessed: 10-Feb-2013].

[16] S. A. Matei and C. Dobrescu, "Wikipedia's 'Neutral Point of View': Settling Conflict through Ambiguity," Inf. Soc., vol. 27, no. 1, pp. 4051, Dec. 2010.

[17] Wikipedia Contributors, "Major depressive disorder," Wikipedia, The Free Encyclopaedia. Wikimedia Foundation, 2014.

[18] A. Brosch, "Hyperbole and a Half: Adventures in depression," 2011. Available: http://hyperboleandahalf.blogspot.com/2011/10/adventuresin-depression.html.

[19] A. Brosch, "Hyperbole and a Half: Depression Part Two," 2013. http://hyperboleandahalf.blogspot.com/2013/05/depression-parttwo.html.

[20] S. Reuter, "Depression," 2012. http://subjectuntitled.tumblr.com/post/43374582693/artdesignwarsylvie-reuter-depression.

[21] Z. Quinn, "Depression Quest," 2013. http://www.depressionquest.com/. 